\title{
The Research on the Absolute Radiometric Calibration of Hyperspectral Camera Based on Reflectance Basis
}

\author{
Zhenjie Yang ${ }^{1,}$, , Kangnian Zhao ${ }^{2, \text { b }}$ \\ ${ }^{1}$ Department of Geological Engineering, Qinghai University, Xining, China \\ ${ }^{1}$ Department of Geological Engineering, Qinghai University, Xining, China \\ a454091615@qq.com, ${ }^{\text {b } 13519755989 @ 139 . c o m}$
}

\begin{abstract}
Keywords: hyperspectral, reflectance-based method, absolute radiometric calibration.
Abstract. The hyperspectral cameras often have hundreds of bands, and their absolute radiometric calibration is the premise for terrain remote sensing applications. Using the hyperspectral camera carried in manned aircraft platform carry out field calibration experiment, and according to the reflectance-based method calibration for 6 different reflectivity targets. Draw the calibration coefficient, and make a reality check for it. The result reveals that the DN value good correlation with actual reflectance, both are greater than $98.5 \%$; Calibration accuracy is around 5\%, meets the demand of follow-up remote sensing applications.
\end{abstract}

\section{Introduction}

Pushbroom imaging spectrometer (PHI) ${ }^{[1]}$ developed by Shanghai Institute of technical physics of the Chinese Academy of Sciences, has excellent technical performance. Its performance parameters, such as band number reached 344 , the spectral resolution up to $4 \sim 7 \mathrm{~nm}$, so that China's hyperspectral research in the international forefront. In order to apply it to the ground object recognition, however we need to make absolute radiometric calibration.

The sensor radiometric calibration technology is an indispensable and important part for applications of remote sensing data quantitative ${ }^{[2]}$; absolute radiometric calibration aim at establishing quantity relationship between digital signal measured by sensor and corresponding radiation energy , and convert an image digital value to a physical quantity, to determine a standard for direct judgment and comparison of objects, and make a quantitative analysis of remote sensing data, so that can better identify objects ${ }^{[3,4]}$; In the outfield usually select dry and clean atmosphere, and open space to do the radiometric calibration for the developed instrument by using the reflect signals of sunlight passing through the reference plate ${ }^{[5]}$.

About the method of the absolute radiometric calibration in the site calibration, there are reflectance-based method, irradiance-based method and radiance-based method ${ }^{[6]}$. There are some research institutes in the United States, Canada, Australia and Japan. They are the most thorough, the longest experimental and the most representative at calibration site in world. Since using BaiSha site to radiometric calibration from 1987 for TM, P.N.Slater, S.F.Biggar ${ }^{[7]}$ and K.J.Thome ${ }^{[8]}$ who are representatives in the remote sensing group of Optical Sciences Center of Arizona University in the US. They get the calibration coefficients of multiple sensors in different periods after succeeded in radiometric calibration based on multiple sensors, such as Landsat-5TM, Landsat-7ETM+, SPOT, MODIS, SeaWiFS, ALI, Hyperion, Ikonos, ASTER, MISR. What compare with the results of a variety of calibration methods, such as pre-launch calibration, calibration on orbiting that proved the effectiveness of the field calibration method. Canada's remote sensing center is responsible for remote sensing radiometric calibration and application in Canada and P.M.Teillet ${ }^{[9,10]}$ is a representative figure. He used airborne hyperspectral sensors to make radiometric calibration for NOAA-14AVHRR, Orbview-2SeaWiFS, SPOT-4VGT, Landsat-5TM and SPOT-2HRV, and good results have been achieved.

The improvement of precision mainly depends on accuracy of calculation mode and the measuring accuracy of each input parameters. On the one hand, it is a good choice to use artificial targets with many kinds of reflectivity as the ground reference. On the other hand, with higher precision of $6 \mathrm{~S}$ 
model or M-COART radiative transfer tool, we can effectively improve the accuracy of radiometric calibration and reduce the errors which caused by stray light of optical remote sensor, out-off-band leakage of filter and Atmospheric model's error.

In this paper, the hyperspectral sensor is placed on a man-machine, and the reflectance-based method is used in the field, and $6 \mathrm{~S}$ radiative transfer model is used in absolute radiometric calibration.

\section{Basic Principles and Methods of Absolute Radiometric Calibration}

The purpose of absolute radiometric calibration is to determine the quantitative relationship between the output voltage of the sensor and the incident absolute light energy. Radiometric correction means that convert $\mathrm{DN}$-value image without any physical meaning to radiation product image with physical meaning, such as reflectivity images and radiance images. The basic transformation model of the $\mathrm{DN}$-value image to the reflectivity (or radiance) image is:

$\rho=a^{*} D N+b$.

$\rho$ : Reflectivity or radiance;

$a$ : absolute calibration coefficient----gain;

$b$ : absolute calibration coefficient----offset;

If $a, b$ both are reflectance calibration coefficients, $\rho$ is corresponding reflectivity product. If $a, b$ both are radiance calibration coefficients, $\rho$ is corresponding radiance product.

Field calibration is to make use of opening and having uniform reflectivity background types to calibrate for the sensor, such as grass, sand (Fig. 1) seawater or reflectivity target.

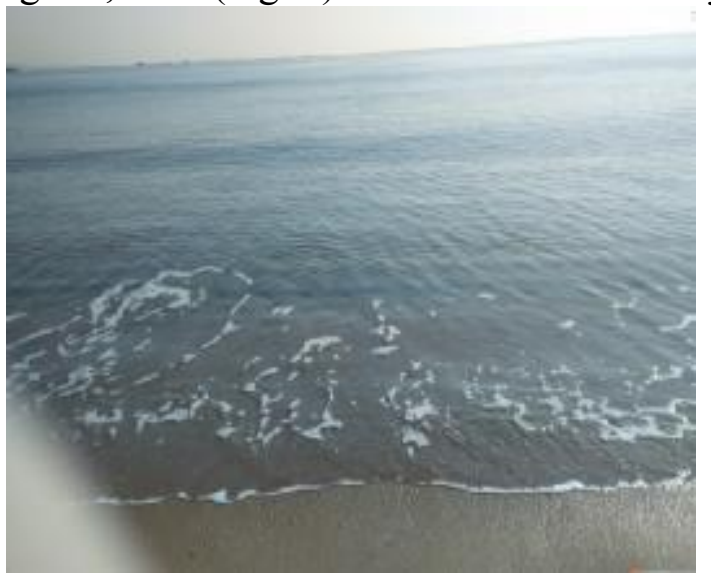

Fig. 1 Sand and seawater in the field

\section{Analysis and Evaluation for Accuracy of Absolute Radiometric Calibration in the Field}

From the visible to near-infrared bands, the feature type with typical reflectivity always selected as the target, and the method of retrieving the target reflectivity by the remote sensing is used to verify the calibration accuracy of each band. For example, if the grassland is chosen as the target, it is necessary that the apparent reflectance value which calculated by calibration coefficients and CCD images compare with the measured inversion spectral reflectance of the grassland when the sensor overhead. And which is used to verify the accuracy of absolute radiometric calibration. On the ground, the reflectivity of the measured grassland is still using the $6 \mathrm{~S}$ radiative transfer model to carry out the inversion and get apparent reflectance, its flow can be represented in Fig. 2. Specific formulas are shown in Table. 1. The relative difference indicates the true accuracy of the absolute radiometric calibration in the field. 


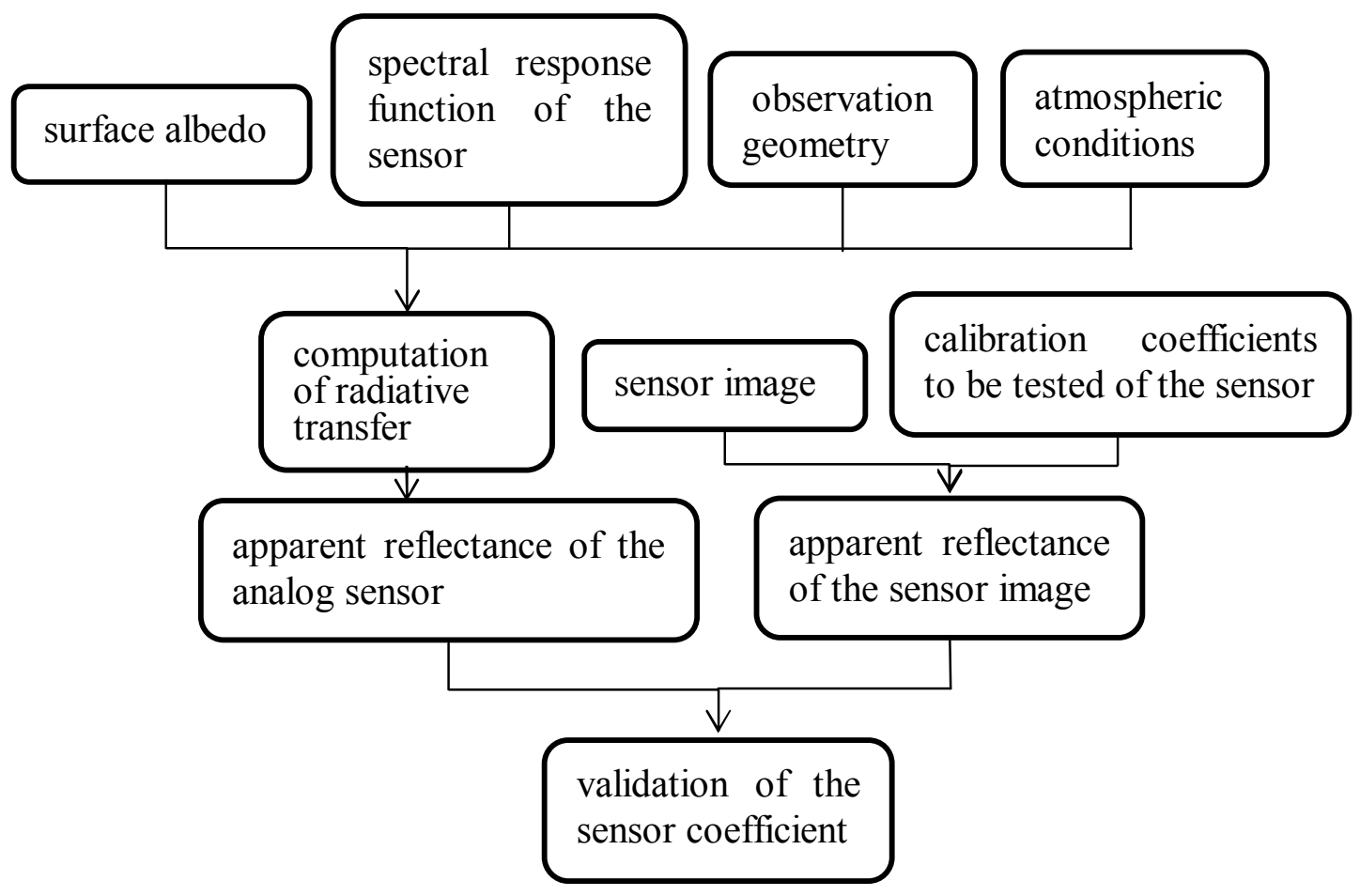

Fig. 2 Process of validation outside

Tab. 1 Calculating table of validation

\begin{tabular}{|c|c|c|c|c|}
\hline & Band1 & Band2 & $\ldots$ & Bandn \\
\hline $\begin{array}{l}\text { The TOA radiance value or } \\
\text { reflectance value of } \\
\text { inversion by the absolute } \\
\text { radiometric calibration } \\
\text { coefficient }\end{array}$ & $L_{1}\left(\rho_{1}\right)$ & & & $L_{n}\left(\rho_{n}\right)$ \\
\hline $\begin{array}{l}\text { The TOA radiance value or } \\
\text { reflectance value of the } \\
\text { measured surface } \\
\text { reflectance inversion }\end{array}$ & $L_{1}^{\prime}\left(\rho_{1}^{\prime}\right)$ & & & $L_{n}^{\prime}\left(\rho_{n}{ }^{\prime}\right)$ \\
\hline Relative difference $[\%]$ & $\frac{\left|L_{1}-L_{1}{ }^{\prime}\right|}{L_{1}}\left(\frac{\left|\rho_{1}-\rho_{1}{ }^{\prime}\right|}{\rho_{1}}\right)$ & & & $\frac{\left|L_{n}-L_{n}{ }^{\prime}\right|}{L_{n}}\left(\frac{\left|\rho_{n}-\rho_{n}{ }^{\prime}\right|}{\rho_{n}}\right)$ \\
\hline
\end{tabular}

\section{Outfield Radiometric Calibration Test}

The UAV remote sensing loading comprehensive verification field in Inner Mongolia Baotou west of $88 \mathrm{~km}$ and elevation is about $995 \mathrm{~m}$. Where was chosen as test site of outfield calibration. Time is selected in October 2013. Six spectral radiance targets (as shown in Fig. 3) was chosen as calibration equipment, they have better lambert and uniform spectrum property, their reflectivity are $4.5 \%, 20 \%$, $30 \%, 40 \%, 50 \%$, and $60 \%$ respectively. There are a small range of uniform grass and sand near the 
target. Flight altitude is about 2000 meters, and the geometric resolution of the hyperspectral image is about $0.5 \mathrm{~m}$.

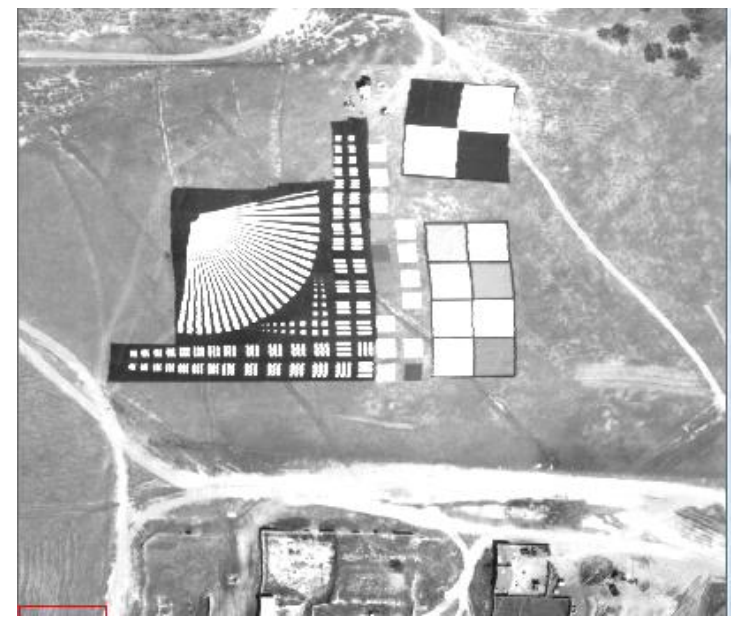

Fig. 3 Ground targets

\section{Calculation of Absolute Calibration Coefficient of External Field}

Calculate the apparent radiance of 6 kinds of radiation level targets in 344 channels at the entrance of the sensor; 20 points are collected on each target and took the average. This average value is the final DN value. The DN value of each channel is shown in Fig. 4. Select 4 channels for description, based on the DN value and apparent radiance, the gain and offset of the calibration coefficient is attained by use of the least square linear regression (Showed in Table. 2).

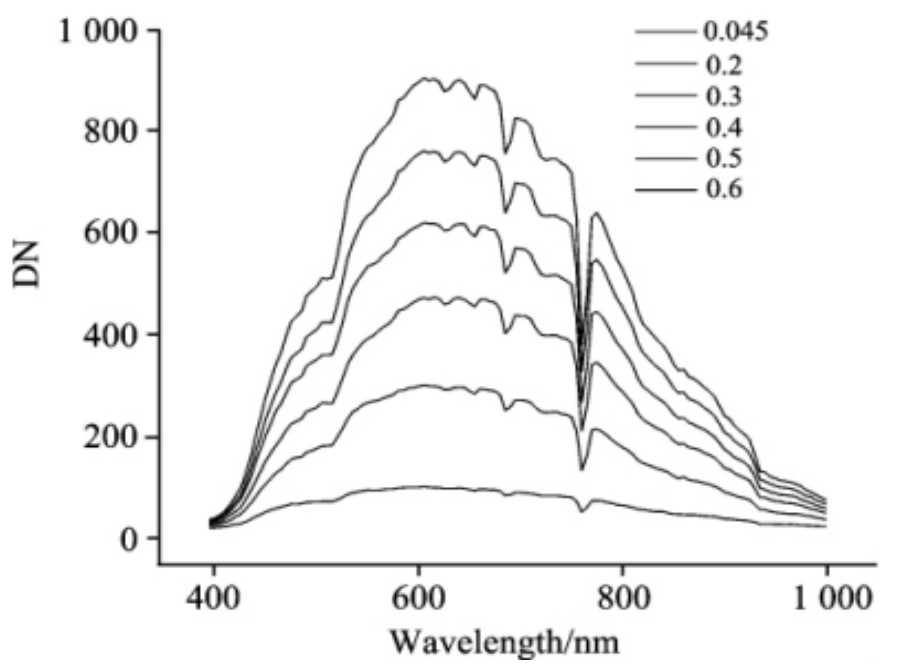

Fig. 4 DN value of six pieces of reflectivity targets in each band

Tab.2 Field's absolute calibration coefficient of band 1 to 4

\begin{tabular}{|l|l|l|l|l|}
\hline Wavelength & Band1 & Band2 & Band3 & Band4 \\
\hline Gain & 1.26580 & 2.003581 & 0.903650 & 1.993706 \\
\hline Bias & 11.8179 & -12.621670 & 23.696127 & 4.072388 \\
\hline
\end{tabular}

The correlation coefficients were calculated for each band by the 6 groups of DN values and the actual reflectance, as shown in Fig. 5. Found that the correlation is very good, which are more than $98.5 \%$. The results show that the hyperspectral camera is better in the linear responsivity and results of the Radiometric Calibration are ideal. 


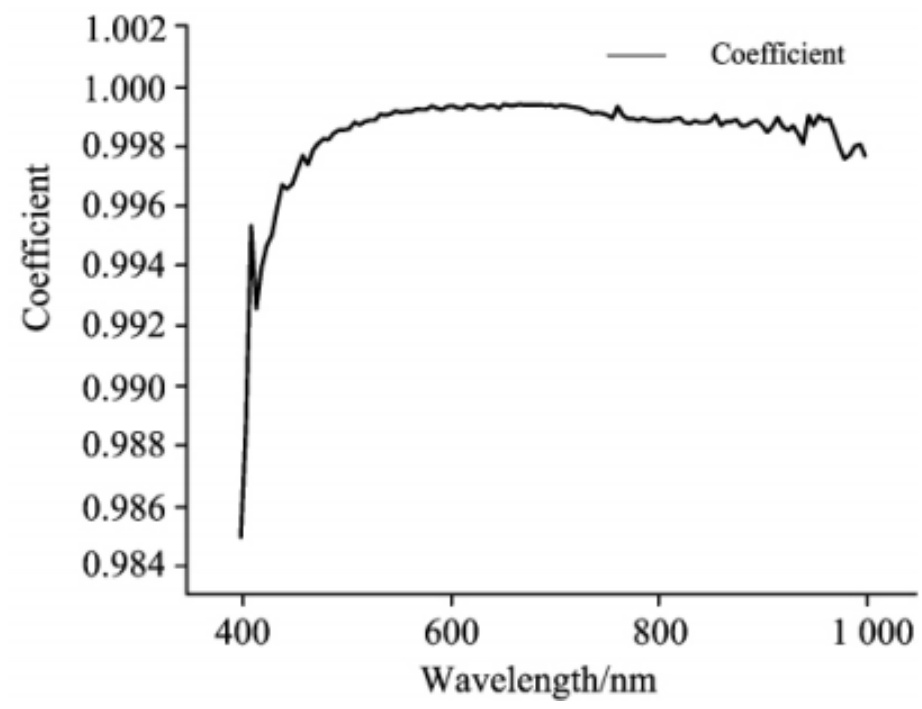

\section{The Results of the Authenticity Test}

Fig.5 Correlation coefficient between $\mathrm{DN}$ value and reflectivity

The time to shoot the image is the next day October 15, 2014, 15:53:06 pm. Select the corresponding aerial image area of three block of concrete nearby and statistic their image gray. Collect the reflectivity of 10 points in each concrete floor and calculate their average value, this average value is used as the average reflectivity of this block of concrete. Finally, the average reflectivity of these three blocks of concrete is treated as measured reflectivity. What the measured reflectivity of the concrete when the aircraft overhead is shown in Fig. 6. By using the $6 \mathrm{~S}$ model to inverse the concrete's reflectivity, the inversion result and the calibration accuracy are shown in Table 3.

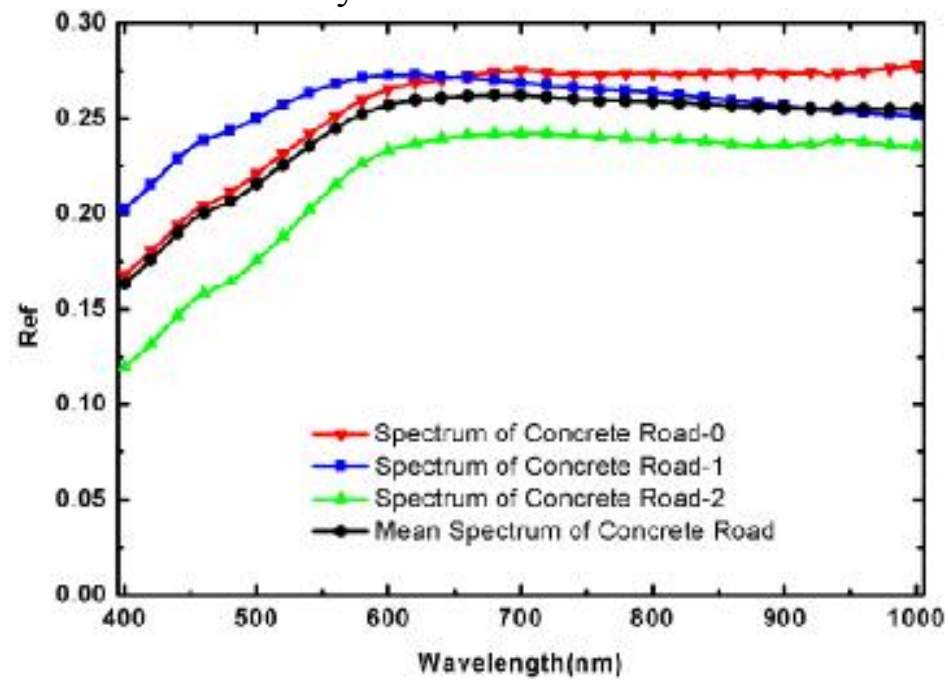

Fig.6 Measured concrete's reflectivity

Tab. 2 Reflectance retrieval's results and the calibration accuracy of concrete

\begin{tabular}{|l|l|l|l|l|}
\hline $\begin{array}{l}\text { DN value of } \\
\text { image }\end{array}$ & $\begin{array}{l}\text { Apparent } \\
\text { radiance }\end{array}$ & $\begin{array}{l}\text { Inversion } \\
\text { reflectivity }\end{array}$ & $\begin{array}{l}\text { Measured } \\
\text { reflectance }\end{array}$ & $\begin{array}{l}\text { Calibration } \\
\text { accuracy }\end{array}$ \\
\hline 76.25 & 108.335 & 0.186 & 0.195 & $4.8 \%$ \\
\hline 79.29 & 145.983 & 0.244 & 0.255 & $4.3 \%$ \\
\hline 87.45 & 102.72 & 0.195 & 0.188 & $3.6 \%$ \\
\hline 71.73 & 147.08 & 0.268 & 0.252 & $5.9 \%$ \\
\hline
\end{tabular}

The accuracy of the inversion are $4.8 \%, 4.3 \%, 3.6 \%, 5.9 \%$ respectively, each is almost the same as the conventional standard (5\%). It proved that the calibration coefficient is correct. This accuracy in the practical application can achieve the ideal requirements. 


\section{Conclusions}

Absolute radiation calibration plays an important role in remote sensing quantitative research. In this paper, what the absolute radiation calibration method based on the reflectivity method is effective used for hyperspectral camera. And which is a key step in the pretreatment phase in application of remote sensing image. And it can also be the basis for the following remote sensing research and experimental analysis.

\section{References}

[1] Shao Hui, Jianyu Wang. Journal of Remote Sensing,1998, 2(4): 251-254. (in Chinese).

[2] Xingfa Gu, Guoliang Tian, Xiaowen Li, et al. Science in China (Series E), 2006, 35(B12): 1-10. (in Chinese).

[3] Qingxi Tong, Bing Zhang, Lanfen Zheng Hyperspectral Remote Sensing. Higher Education Press. (in Chinese).

[4] Yini Duan, Lifu Zhang, Lei Yan, et al. Journal of Remote Sensing, 2014, 18(3):597-617. (in Chinese).

[5] Fuchun Chen, Guilin Chen. Chinese Journal of Quantum Electronics, 2007. FY-2C 24(6): 709 713 (in Chinese).

[6] Slater P N, Biggar S F, Holm R G, et al. Remote Sensing of Environment, 1987, 22:11-37.

[7] Biggar S F, Dinguirard M C, Gellman D I, et al. Radiometric calibration of SPOT 2 HRV: $a$ comparison of three methods. Calibration of Passive Remote Observing Optical \& Microwave Instrumentation. International Society for Optics and Photonics, 1991.

[8] Thome K J. Remote Sensing of Environment, 2001, 78:27-38.

[9] Teillet P M, Fedosejevs G, Thome K J, et al. Remote Sensing of Environment, 2007, 110(3):393-409.

[10]Teillet P M, Barker J L, Markham B L, et al. Remote Sensing of Environment, 2001, 78:39-54. 